${ }^{1}$ Federal University Pelotas - UFPel School of Dentistry, Department of Periodontology, Pelotas, RS, Brazil.

${ }^{2}$ School of Pharmacy, Dentistry and Nursing, Federal University of Ceará, Fortaleza, Brazil.

${ }^{3}$ School of Dentistry, Unichristus University, Fortaleza, Brazil.

4School of Pharmacy, Dentistry and Nursing, Periodontology Department, Federal University of Ceará, Fortaleza, Brazil.
Corresponding author:

Francisco Wilker M G Muniz (Professor, Federal University Pelotas, Pelotas, Brazil. Rua Gonçalves Chaves, 457, Pelotas, Rio Grande do Sul, Brazil. Zip code: 96015-560. +5585996520187 wilkermustafa@gmail.com.

Received: June 06, 2018 Accepted: November 18, 2018

\section{Stressors, psychological well-being, and overall health amongst students from public and private dental schools}

\author{
Francisco Wilker Mustafa Gomes Muniz'1, Marcelo \\ Bruno Lemos de Oliveira², Isadora Daniel Barros², \\ Patrícia Maria Costa de Oliveira ${ }^{3}$, Lidiany Karla \\ Azevedo Rodrigues ${ }^{4}$, Rosimary de Sousa Carvalho ${ }^{4}$
}

Aim: This study aimed to correlate stressors with psychological well-being and health factors in dental students from public and private schools. Methods: From February to May 2015, three different instruments (Dental Environment Stress - DES Psychological General Well-Being - PGWB - and SF-36 Health Survey) were applied to students from two public and two private dental schools from the State of Ceará, Brazil. MannWhitney test or $t$ test for independent samples were used in order to compare the stressors between private and public dental schools students. Correlations to each DES domain were performed using Kendall's Tau C test. Results: A total of 92 (45.32\%) and 111 (56.68\%) students from public and private schools, respectively, answered the questionnaire. Students from public schools demonstrated significantly higher scores in DES/academic performance and DES/personal and institutional factors $(p<0.05)$. Significant negative correlations were detected between PGWB/anxiety and PGWB/general with all DES domains for both public and private schools ( $p<0.05)$. Additionally, DES/ academic performance was significantly correlated with several SF-36 domains, such as physical function, vitality, and social functioning, to both public and private schools $(p<0.05)$. However, DES/academic performance and SF-36/role physical was only significantly correlated in private school students $(r=-0.171$, $p=0.039$ ), while SF-36/bodily pain ( $r=-0.274, p<0.001)$, general health $(r=-0.245, p=0.001)$, and mental health $(r=-0.286, p<0.001)$ were significantly correlated with DES/academic performance only in public school students. Conclusion: Students from public and private dental schools presented different stressor patterns. Additionally, most of DES domains were significantly associated with PGWB and SF-36 to both public and private schools.

Keywords: Stress, psychological. Students, dental. Health personnel. 


\section{Introduction}

Psychological stress is defined as a dynamic process, as the perceived environmental pressure which jeopardizes an individual's well-being ${ }^{1}$. It is well known that university students can be highly stressed due to education demand ${ }^{2}$. This may lead to emotional, psychological, and physical impairments, which may compromise their education and general health ${ }^{3}$.

Several studies have identified the sources of stress in dental undergraduate students' behavior. The literature has shown some contributory stressful factors, such as management of patients' schedules, uncooperative patients, decreased time to relax, pressure to fulfill all technical and scientific demands, and fear of failing a school period ${ }^{4,5}$. It has been also demonstrated that, in comparison to the general population, dental students presented stressors in higher levels ${ }^{6}$, especially due to the highly technical tasks required ${ }^{5}$.

In Brazil, undergraduation in Dentistry is offered by several educational institutions, of which 56 and 176 were public and private dental schools, respectively, in $2015^{7}$. Nevertheless, few studies have assessed the stressors in Brazilian dental students ${ }^{8,9}$. Both types of schools demand a selective process, as entry requirement. However, the entrance exams in public dental schools is highly competitive.

Considering that private and public dental schools present different profiles of student population, the rationality for comparing both institutions is of utmost importance, mainly when one considers that several strategies may be developed in order to face health problems in these distinct populations. Therefore, this study aimed to analyze the stressors, overall health, psychological well-being and its correlation with stressors in dental students from public and private dental schools from the State of Ceará, Brazil. The null hypothesis of this study is that there are no significant differences in the stressors between students from public and private dental schools.

\section{MATERIAL AND METHODS}

\section{Study Type and Ethical Aspects}

This is a cross-sectional study that included dental students from four different dental schools from Ceará, Brazil. The study protocol was approved by the Institutional Review of the Federal University of Ceará under protocol 953.335/2015, and all volunteers signed an informed consent. The study was conducted in full accordance with the World Medical Association Declaration of Helsinki.

\section{Sample Source}

The chosen public dental schools are from Universidade Federal do Ceará, one at the Campus of Fortaleza (State capital of Ceará) and the other at the Sobral Campus, (the fifth largest city of Ceará with approximately 203,000 inhabitants), as well as the private schools from Universidade de Fortaleza (UNIFOR) based in the city of Fortaleza, and from Faculdade Católica Rainha do Sertão (FCRS) in Quixadá (a city of Ceará with approximately 86,000 inhabitants). In 2015, a total of 519 and 981 students were regularly enrolled in the public and private dental schools, respectively. To be included, participants had to be 
at least 18 years of age and be regularly enrolled in 2015, attending any academic term. Additionally, they had to complete at least the following variables in the questionnaire: sex, academic term, University/Faculty attended. Students that answered all these variables, but did not answer all the three instruments, were excluded from data analysis.

All dental schools involved in the present study have a closed group on Facebook, and each class from each dental school have an e-mail managed only by the class students. From February to May 2015, invitations were sent to these social media groups and to these e-mails, explaining the main objectives and the inclusion criteria of the present study. The volunteers were encouraged to answer the invitation by sending their personal e-mail to one of the researchers involved in the present study. They could also make an appointment to answer the questionnaire in a printed format. The confirmation of the student enrollment in the year 2015 was assured by contacting each dental school dean.

\section{Study Power}

As all dental students have been invited to participate, a sample size calculation was not previously performed. The scores in Dental Environment Stress (DES) instrument was considered the main outcome of this study. Therefore, a study power was estimate, using the following parameters: alpha of $5 \%$, mean \pm SD DES total scores of $2.460 \pm 0.505$ and $2.257 \pm 0.543$ to public and private dental schools, respectively. A power of $78.67 \%$ for a two-sample comparison of means was determined. To achieve $80 \%$ power in this study, 209 dental students were necessary, which is closer to the final sample size of 203 participants.

\section{Instruments Applied and Collected Variables}

Three different instruments were used, DES, the Psychological General Well-Being (PGWB) index, and the Medical Outcomes Study 36-Item Short-Form Health Survey (SF-36). Both DES and SF-36 were translated and validated to a Brazilian sample ${ }^{8,10}$. The PGWB instrument was previously validated to a Portuguese sample ${ }^{11}$ and another study had used it in Brazilian samples ${ }^{12}$. In addition to the three instruments, sex (male or female), age, clinical training (clinical training or pre-clinical training courses), dental school attended (UFC/Fortaleza, UFC/Sobral, UNIFOR or FCRS) were collected in this questionnaire.

The Brazilian validated format of the DES instrument is constituted of 36 questions, which identify and quantify the sources of stress in dental students (8). The items were answered based on the four point Likert scale, such as not stressful (1 point), slightly stressful (2 points), moderately stressful (3 points), and very stressful (4 points). Higher scores mean a more stressful source. This instrument is divided into five dimensions.

The PGWB index measures the subjective general well-being and distress in the dental students. The original scoring per item ranges from 0 to 5 , giving a maximum score of 110 . This instrument is composed by 22 questions and includes six dimensions. The SF-36 is a multidimensional instrument composed of 36 items and provides composite scores for eight dimensions. Lower scores mean a less favorable health state.

\section{Dealing With Missing Data}

To each instrument, different approaches were performed in order to deal with missing data. For PGWB, as the missing data were low, the items were replaced by employ- 
ing inter-item correlations, as previously reported ${ }^{13}$. For the other instruments, when a score item was absent, the mean scores of the dimension was calculated and attributed to the missing question.

\section{Statistical Analysis}

Public dental schools were compared to the private dental schools. Shapiro-Wilk normality test was applied to each variable, and when a normal distribution was detected, $t$ test for independent samples was applied. Mann-Whitney test was used only when a non-normal distribution was detected. The comparisons of sex and attending to academic clinical courses were performed by Chi-square. The reliability of the three instruments was assessed by Cronbach's alpha coefficients.

Additionally, Cohen's d assessed the effect size of all comparisons performed. Kendall's Tau C test coefficients were used to assess the correlation between DES dimensions and the dimensions of the two other instruments.

The statistical analysis was carried out with SPSS version 21.0 for Windows (IBM®) SPSS Statistics, New York, USA), and a p-value $<0.05$ was considered to represent a significant difference. Bonferroni correction was applied in the analyses of each DES domain, as each question had already been included in the domains. Therefore, the new $p$-values established were: academic performance, $p<0.005$; difficulties and insecurities about their professional future, $p<0.008$; responsibilities with patients, $p<0.013$; Personal and institutional factors, $p<0.006$; Interpersonal relationships, $p<0.006$.

\section{RESULTS}

Overall, 237 e-mails were received, and the questionnaire was sent to 231 dental students, as six of them were not regularly enrolled in the year 2015. Two-thousand and three students returned the questionnaire (response rate of $87.88 \%$ ). Ninety-eighty (48.28\%) and 105 (51.72\%) questionnaires were, respectively, filled in a printed and electronic format. Table 1 shows the demographical characteristics, according to dental school type. Students from public dental schools were significantly older in comparison to the ones from private schools $(p=0.003)$.

\section{DES Instrument}

The overall and each dimension reliability analyses found for DES instrument are demonstrated in Table 2. The stressors, according to dental school type, are expressed in Table 3. Public dental school students presented significantly higher total scores in the DES instrument in comparison to private dental school students $(p=0.005)$. The effect size was considered small to the overall score (Cohen's $d=0.381$ ), other questions showed medium to large effect size, such as amount of assigned classwork (Cohen's $d=0.553$ ), atmosphere created by faculty (Cohen's $d=0.932$ ), lack of time for relaxation and recreation (Cohen's $d=0.647$ ), completing graduation requirements (Cohen's $d=0.523$ ), expectations of dental school and the reality (Cohen's $d=0.596$ ), and lack of time to do assigned school work (Cohen's $d=0.577$ ). To all those questions, students from public dental schools presented higher scores. 
Table 1. Sample demographical characteristics, according the dental school type.

\begin{tabular}{|c|c|c|c|c|c|}
\hline & & $\begin{array}{l}\text { Overall } \\
(n=203)\end{array}$ & $\begin{array}{c}\text { Public } \\
(n=92 ; \\
45.32 \%)\end{array}$ & $\begin{array}{l}\text { Private } \\
(n=111 ; \\
56.68 \%)\end{array}$ & P-value \\
\hline Dental School & $\begin{array}{c}\text { UFC - Fortaleza }-n(\%) \\
\text { UFC - Sobral }-n(\%) \\
\text { UNIFOR }-n(\%) \\
\text { FCRS }-n(\%)\end{array}$ & - & $\begin{array}{c}65(70.65) \\
27(29.35) \\
- \\
-\end{array}$ & $\begin{array}{c}- \\
- \\
90(81.08) \\
21(18.92)\end{array}$ & - \\
\hline Sex & $\begin{array}{c}\text { Male - n (\%) } \\
\text { Female - n (\%) }\end{array}$ & $\begin{array}{c}59(29.1) \\
144(70.9)\end{array}$ & $\begin{array}{l}30(32.6) \\
62(67.4)\end{array}$ & $\begin{array}{l}29(26.1) \\
82(73.9)\end{array}$ & $0.353^{*}$ \\
\hline Age (in years) & $\begin{array}{c}\text { Mean } \pm S D \\
\text { (median-min.;max.) }\end{array}$ & $\begin{array}{c}21.15 \pm 4.70 \\
(21-18 ; 50)\end{array}$ & $\begin{array}{c}22.31 \pm 2.75 \\
(22-18 ; 30)\end{array}$ & $\begin{array}{c}22.01 \pm 5.82 \\
(20-18 ; 50)\end{array}$ & $0.003 a \S$ \\
\hline Clinical training & $\begin{array}{l}\text { Pre-clinical training }-\mathrm{n}(\%) \\
\text { Clinical training }-\mathrm{n}(\%)\end{array}$ & $\begin{array}{l}75(36.9) \\
128(63.1)\end{array}$ & $\begin{array}{l}36(39.1) \\
56(60.9)\end{array}$ & $\begin{array}{l}39(35.1) \\
72(64.9)\end{array}$ & $0.563^{*}$ \\
\hline
\end{tabular}

Legend: UFC: Universidade Federal do Ceará; UNIFOR: Universidade de Fortaleza; FCRS: Faculdade Católica

Rainha do Sertão; *Chi-square; aMann-Whitney test; §ुCohen's d=0.066

Table 2. Cronbach's alpha of all domain from the 3 questionnaires used.

\begin{tabular}{|c|c|}
\hline Domain & Cronbach's alpha \\
\hline \multicolumn{2}{|l|}{ DES } \\
\hline Academic performance & 0.802 \\
\hline Difficulties and insecurities about their professional future & 0.822 \\
\hline Responsibilities with patients & 0.755 \\
\hline Personal and institutional factors & 0.751 \\
\hline Interpersonal relationships & 0.679 \\
\hline Overall & 0.915 \\
\hline \multicolumn{2}{|l|}{ PGWBI } \\
\hline Anxiety & 0.756 \\
\hline Depressed mood & 0.665 \\
\hline Positive well-being & 0.563 \\
\hline Self-control & 0.411 \\
\hline General health & 0.540 \\
\hline Vitality & 0.521 \\
\hline Overall & 0.868 \\
\hline \multicolumn{2}{|l|}{ SF-36 Health Survey } \\
\hline Physical functioning & 0.823 \\
\hline Role-physical & 0.622 \\
\hline Bodily pain & 0.710 \\
\hline General health & 0.677 \\
\hline Vitality & 0.683 \\
\hline Social functioning & 0.373 \\
\hline Role-emotional & 0.639 \\
\hline Mental Health & 0.711 \\
\hline Overall & 0.831 \\
\hline
\end{tabular}


Table 3. Mean (SD) stressors by type of school, using DES scale.

\begin{tabular}{|c|c|c|c|c|c|}
\hline & $\begin{array}{l}\text { Overall } \\
(n=203)\end{array}$ & $\begin{array}{l}\text { Public } \\
(n=92)\end{array}$ & $\begin{array}{l}\text { Private } \\
(n=111)\end{array}$ & $\begin{array}{l}\text { Difference } \\
\text { between } \\
\text { school type } \\
(\mathrm{p} \text {-value)* }\end{array}$ & Cohen's d \\
\hline 1: Amount of assigned classwork & $2.59(1.00)$ & $2.88(0.88)$ & $2.35(1.03)$ & $<0.001$ & 0.553 \\
\hline $\begin{array}{l}\text { 2: Lack of cooperation by patient in } \\
\text { their home care }\end{array}$ & $1.97(0.94)$ & $1.92(0.94)$ & $2.00(0.94)$ & 0.872 & 0.085 \\
\hline 3: Difficulty of classwork & $2.31(0.92)$ & $2.52(0.93)$ & $2.14(0.88)$ & 0.005 & 0.420 \\
\hline $\begin{array}{l}\text { 4: Responsibilities for } \\
\text { comprehensive patient care }\end{array}$ & $1.89(1.08)$ & $1.92(1.07)$ & $1.87(1.09)$ & 0.173 & 0.046 \\
\hline 5: Competition for grades & $2.17(1.16)$ & $2.23(1.21)$ & $2.13(1.12)$ & 0.543 & 0.086 \\
\hline $\begin{array}{l}\text { 6: Patients being late or not } \\
\text { showing for their appointments }\end{array}$ & $2.30(1.22)$ & $2.16(1.26)$ & $2.41(1.19)$ & 0.249 & 0.204 \\
\hline 7: Examinations and grades & $3.48(0.82)$ & $3.61(0.71)$ & $3.37(0.88)$ & 0.048 & 0.300 \\
\hline $\begin{array}{l}\text { 8: Difficulty in learning clinical } \\
\text { procedures }\end{array}$ & $1.99(1.07)$ & $2.03(1.09)$ & $1.95(1.05)$ & 0.004 & 0.075 \\
\hline 9: Atmosphere created by faculty & $2.17(1.17)$ & $2.71(1.12)$ & $1.72(1.00)$ & $<0.001$ & 0.932 \\
\hline $\begin{array}{l}\text { 10: Relations with member of the } \\
\text { opposite sex }\end{array}$ & $1.38(0.82)$ & $1.26(0.69)$ & $1.49(0.90)$ & 0.022 & 0.289 \\
\hline 11: Receiving criticism about work & $2.30(1.04)$ & $2.44(1.05)$ & $2.19(1.02)$ & 0.102 & 0.242 \\
\hline $\begin{array}{l}\text { 12: Difficulty in learning precision } \\
\text { manual skills required in preclinical } \\
\text { and laboratory work }\end{array}$ & $2.01(1.10)$ & $2.16(1.19)$ & $1.88(1.00)$ & 0.001 & 0.255 \\
\hline $\begin{array}{l}\text { 13: Lack of self-confidence in be a } \\
\text { successful dental student }\end{array}$ & $2.44(1.15)$ & $2.66(1.12)$ & $2.25(1.14)$ & 0.010 & 0.363 \\
\hline $\begin{array}{l}\text { 14: Lack of self-confidence in be a } \\
\text { successful dentist }\end{array}$ & $2.55(1.13)$ & $2.72(1.15)$ & $2.41(1.09)$ & 0.050 & 0.277 \\
\hline $\begin{array}{l}\text { 15: Lack of time for relaxation and } \\
\text { recreation }\end{array}$ & $3.11(1.03)$ & $3.45(0.88)$ & $2.82(1.06)$ & $<0.001$ & 0.647 \\
\hline $\begin{array}{l}\text { 16: Amount of cheating in dental } \\
\text { school }\end{array}$ & $2.22(1.10)$ & $2.20(1.14)$ & $2.24(1.06)$ & 0.636 & 0.037 \\
\hline 17: School rules and regulations & $2.25(1.02)$ & $2.47(1.02)$ & $2.06(0.99)$ & 0.007 & 0.408 \\
\hline $\begin{array}{l}\text { 18: Working on patients with dirty } \\
\text { mouths }\end{array}$ & $2.01(1.11)$ & $2.05(1.12)$ & $1.97(1.10)$ & 0.034 & 0.072 \\
\hline $\begin{array}{l}\text { 19: Lack of family atmosphere in } \\
\text { the dormitories during school }\end{array}$ & $1.81(1.11)$ & $1.80(1.11)$ & $1.82(1.11)$ & 0.815 & 0.018 \\
\hline $\begin{array}{l}\text { 20: Completing graduation } \\
\text { requirements }\end{array}$ & $2.85(1.21)$ & $3.19(1.04)$ & $2.58(1.28)$ & 0.001 & 0.523 \\
\hline $\begin{array}{l}\text { 21: Reconcile personal life issues } \\
\text { with dental school routines }\end{array}$ & $3.03(1.01)$ & $3.27(0.92)$ & $2.83(1.05)$ & 0.002 & 0.446 \\
\hline $\begin{array}{l}\text { 22: Expectations of dental school } \\
\text { and the reality }\end{array}$ & $2.50(1.08)$ & $2.84(1.03)$ & $2.22(1.05)$ & $<0.001$ & 0.596 \\
\hline $\begin{array}{l}\text { 23: Lack of participation in the } \\
\text { school's decision-making }\end{array}$ & $2.31(1.12)$ & $2.51(1.12)$ & $2.15(1.09)$ & 0.020 & 0.326 \\
\hline 24: Fear of failing course or year & $3.34(1.02)$ & $3.59(0.74)$ & $3.13(1.16)$ & 0.009 & 0.473 \\
\hline $\begin{array}{l}25 \text { : Insecurity concerning } \\
\text { professional future }\end{array}$ & $2.87(1.01)$ & $2.95(0.96)$ & $2.80(1.05)$ & 0.335 & 0.149 \\
\hline 26: Financial responsibilities & $2.97(1.09)$ & $3.12(1.02)$ & $2.85(1.14)$ & 0.086 & 0.250 \\
\hline $\begin{array}{l}\text { 27: Lack of time to do assigned } \\
\text { school work }\end{array}$ & $2.97(0.96)$ & $3.26(0.78)$ & $2.73(1.04)$ & $<0.001$ & 0.577 \\
\hline Continue & & & & & \\
\hline
\end{tabular}




\begin{tabular}{|c|c|c|c|c|c|}
\hline \multicolumn{6}{|l|}{ Continuation } \\
\hline $\begin{array}{l}\text { 28: Considering entering some } \\
\text { other filed of work }\end{array}$ & $2.02(1.14)$ & $1.99(1.15)$ & $2.05(1.14)$ & 0.668 & 0.052 \\
\hline $\begin{array}{l}\text { 29: Difficulty in undertake conjugal } \\
\text { commitments }\end{array}$ & $2.10(1.21)$ & $2.13(1.19)$ & $2.08(1.23)$ & 0.768 & 0.041 \\
\hline 30: Personal physical health & $2.46(1.17)$ & $2.72(1.21)$ & $2.25(1.09)$ & 0.005 & 0.408 \\
\hline $\begin{array}{l}\text { 31: Attitudes of school toward } \\
\text { women dental students }\end{array}$ & $1.68(1.02)$ & $1.46(0.79)$ & $1.86(1.15)$ & 0.011 & 0.405 \\
\hline $\begin{array}{l}\text { 32: Family conflict throughout your } \\
\text { career development }\end{array}$ & $1.77(1.09)$ & $1.70(1.01)$ & $1.84(1.15)$ & 0.556 & 0.129 \\
\hline $\begin{array}{l}\text { 33: Discrimination due to race, } \\
\text { class status or ethnic group }\end{array}$ & $1.70(1.01)$ & $1.51(0.88)$ & $1.87(1.08)$ & 0.019 & 0.365 \\
\hline $\begin{array}{l}34 \text { : Inconsistency feedback of your } \\
\text { work between different instructors }\end{array}$ & $2.41(1.20)$ & $2.66(1.14)$ & $2.20(1.21)$ & 0.005 & 0.391 \\
\hline $\begin{array}{l}\text { 35: Fear of being unable to catch } \\
\text { up if get behind }\end{array}$ & $2.82(1.14)$ & $3.01(1.08)$ & $2.66(1.18)$ & 0.029 & 0.309 \\
\hline $\begin{array}{l}\text { 36: Attitudes of school towards } \\
\text { homosexual dental students }\end{array}$ & $1.77(1.08)$ & $1.53(0.93)$ & $1.96(1.15)$ & 0.005 & 0.411 \\
\hline Total mean score & $2.34(0.53)$ & $2.46(0.51)$ & $2.26(0.54)$ & 0.005 & 0.381 \\
\hline
\end{tabular}

Legend: SD - standard deviation; * Mann-Whitney test; Bold numbers mean statistically significance.

Table 4 shows the comparison between dental school type and dimensions to each instrument. Academic performance and personal and institutional factors were significantly higher stressors in public dental school students $(p<0.001$ and $p=0.001$, respectively). However, the only dimension presenting a large effect size was academic performance (Cohen's $d=0.904$ ).

\section{PGWB Index}

The reliability analysis found an alpha coefficient of 0.868 for PGWB index (Table 2). In general, public dental school students presented significantly lower total scores when compared to students from private schools $(p=0.023)$. Additionally, anxiety, positive well-being, and vitality dimensions were significantly lower in these students. Nonetheless, depressed mood, self-control, and general health dimensions did not demonstrate significant difference between dental school type. Vitality was the only dimension showing at least a medium effect size (Cohen's $d=0.909$ ).

\section{SF-36 Health Survey}

The reliability analysis found an alpha coefficient of 0.831 for SF-36 Health survey (Table 2). The total score did not show statistically significance between groups $(p=0.259)$. Similarly, six dimensions were not significantly different between dental school type. On the other hand, two dimensions were significantly lower in the public dental school students, vitality and social functioning ( $p=0.016$ and $p=0.006$, respectively). However, none of the dimensions showed a medium or large effect size.

\section{Correlations of Survey - DES and PGWB}

The correlation between DES and PGWB total scores showed a negative significant correlation to both dental schools type $(r=-0.412$ and $r=-0.386, p<0.001$ to public dental and 
Table 4. Respondents' mean score (SD) on dimensions of each survey by school type.

\begin{tabular}{|c|c|c|c|c|c|}
\hline Dimension & $\begin{array}{l}\text { Overall } \\
(n=203)\end{array}$ & $\begin{array}{l}\text { Public } \\
(\mathrm{n}=92)\end{array}$ & $\begin{array}{l}\text { Private } \\
(\mathrm{n}=111)\end{array}$ & $\begin{array}{l}\text { Difference } \\
\text { between } \\
\text { school type } \\
(\mathrm{p} \text {-value)* }\end{array}$ & Cohen's d \\
\hline \multicolumn{6}{|l|}{ DES } \\
\hline $\begin{array}{l}\text { Academic performance } \\
(10-40)\end{array}$ & $28.15(6.14)$ & 30.91 (5.38) & $25.86(5.79)$ & $<0.001$ & 0.904 \\
\hline $\begin{array}{l}\text { Difficulties and insecurities } \\
\text { about their professional } \\
\text { future }(6-24)\end{array}$ & $14.71(4.94)$ & $15.47(5.01)$ & $14.08(4.81)$ & 0.061 & 0.283 \\
\hline $\begin{array}{l}\text { Responsibilities with patients } \\
(4-16)\end{array}$ & $8.16(3.32)$ & $8.07(3.41)$ & $8.24(3.26)$ & 0.716 & 0.051 \\
\hline $\begin{array}{l}\text { Personal and institutional } \\
\text { factors (8-32) }\end{array}$ & $19.04(5.46)$ & $20.43(5.18)$ & $17.87(5.43)$ & 0.001 & 0.482 \\
\hline $\begin{array}{l}\text { Interpersonal relationships } \\
(8-32)\end{array}$ & $14.52(4.67)$ & $13.68(4.26)$ & $15.21(4.90)$ & 0.022 & 0.294 \\
\hline Total score (36-144) & $84.57(19.25)$ & $88.57(18.20)$ & $81.26(19.55)$ & $0.007 \S$ & 0.387 \\
\hline \multicolumn{6}{|l|}{ PGWB } \\
\hline Anxiety $(0-25)$ & $12.14(4.56)$ & $11.23(4.82)$ & $12.90(4.21)$ & 0.017 & 0.369 \\
\hline Depressed mood (0-15) & $9.73(3.06)$ & $9.65(2.99)$ & $9.80(3.13)$ & 0.834 & 0.049 \\
\hline Positive well-being (0-20) & $10.78(3.15)$ & $10.00(3.07)$ & $11.42(3.08)$ & 0.004 & 0.462 \\
\hline Self-control (0-15) & $9.47(2.66)$ & $9.49(2.74)$ & $9.46(2.61)$ & 0.897 & 0.011 \\
\hline General health $(0-15)$ & $8.66(2.64)$ & $8.51(2.69)$ & $8.78(2.60)$ & 0.507 & 0.104 \\
\hline Vitality $(0-20)$ & $9.83(2.97)$ & $8.96(2.86)$ & $11.56(2.86)$ & $<0.001$ & 0.909 \\
\hline Total score (0-110) & $60.62(14.12)$ & $57.84(14.58)$ & $62.93(13.36)$ & $0.011 \S$ & 0.364 \\
\hline \multicolumn{6}{|l|}{ SF-36 } \\
\hline Physical functioning (0-100) & $74.66(20.32)$ & $77.12(19.95)$ & $72.61(20.49)$ & 0.104 & 0.223 \\
\hline Role physical (0-100) & $43.32(33.62)$ & $42.39(35.69)$ & $44.09(31.93)$ & 0.615 & 0.050 \\
\hline Bodily pain (0-100) & $65.90(21.55)$ & $64.88(22.42)$ & $66.76(20.85)$ & 0.444 & 0.087 \\
\hline General health $(0-100)$ & $61.29(18.14)$ & $60.57(20.50)$ & $61.89(15.97)$ & 0.973 & 0.072 \\
\hline Vitality $(0-100)$ & $46.61(17.89)$ & $43.37(18.41)$ & $49.31(17.05)$ & 0.016 & 0.335 \\
\hline Social functioning $(0-100)$ & $61.01(21.44)$ & $56.79(22.88)$ & 64.55 (19.57) & 0.006 & 0.365 \\
\hline Role emotional (0-100) & $51.65(37.77)$ & $51.09(40.28)$ & $52.12(35.70)$ & 0.895 & 0.027 \\
\hline Mental health $(0-100)$ & $59.55(17.26)$ & $58.43(17.19)$ & $60.47(17.34)$ & 0.506 & 0.118 \\
\hline Total score $(0-800)$ & $463.85(112.02)$ & $454.64(124.52)$ & $471.56(100.32)$ & $0.295 \S$ & 0.150 \\
\hline
\end{tabular}

private dental school students, respectively). Table 5 shows the correlation between each dimension of DES and PGWB surveys according to dental school type. All the significant correlation found were negative, demonstrating lower quality of life while stressors increase. Moreover, these correlations have ranged from low to moderate.

\section{Correlations of Survey - DES and SF-36}

The correlation between DES and SF-36 total scores also showed a negative significant correlation to both dental schools type $(r=-0.260$ and $r=-0.286, p<0.001$ to public dental and private dental school students, respectively). The correlation 
Table 5. Correlations of DES and PGWB dimensions scores, according to dental school type. Correlations are express using Kendall's Tau C test.

\begin{tabular}{|c|c|c|c|c|c|c|c|c|c|c|}
\hline & \multicolumn{2}{|c|}{$\begin{array}{l}\text { DES/Academic } \\
\text { performance }\end{array}$} & \multicolumn{2}{|c|}{$\begin{array}{c}\text { DES/Difficulties } \\
\text { and insecurities } \\
\text { about professional } \\
\text { future }\end{array}$} & \multicolumn{2}{|c|}{$\begin{array}{c}\text { DES/ } \\
\text { Responsibilities } \\
\text { with patients }\end{array}$} & \multicolumn{2}{|c|}{$\begin{array}{c}\text { DES/ } \\
\text { Personal and } \\
\text { institutional } \\
\text { factors }\end{array}$} & \multicolumn{2}{|c|}{$\begin{array}{l}\text { DES/ } \\
\text { Interpersonal } \\
\text { relationships }\end{array}$} \\
\hline & Public & Private & Public & Private & Public & Private & Public & Private & Public & Private \\
\hline PGWB/Anxiety & $-0.468 *$ & $-0.257^{\star}$ & $-0.433^{*}$ & $-0.248 *$ & $-0.198 *$ & $-0.227^{*}$ & $-0.343^{\star}$ & $-0.304^{*}$ & $-0.211^{*}$ & $-0.259 *$ \\
\hline $\begin{array}{l}\text { PGWB/ } \\
\text { Depressed mood }\end{array}$ & $-0.275^{*}$ & $-0.186^{*}$ & -0.230 * & $-0.224^{*}$ & -0.131 & $-0.234^{*}$ & $-0.307^{*}$ & $-0.283^{*}$ & $-0.242^{*}$ & $-0.327^{\star}$ \\
\hline $\begin{array}{l}\text { PGWB/Positive } \\
\text { well-being }\end{array}$ & $-0.205^{\star}$ & $-0.185^{\star}$ & $-0.158^{*}$ & $-0.178 *$ & -0.032 & -0.074 & $-0.143^{*}$ & $-0.255^{\star}$ & $-0.132^{*}$ & $-0.217^{\star}$ \\
\hline PGWB/Self-control & $-0.199 *$ & $-0.219 *$ & $-0.209 *$ & $-0.216^{*}$ & -0.052 & -0.091 & $-0.217^{*}$ & $-0.294^{*}$ & $-0.235^{\star}$ & -0.129 \\
\hline $\begin{array}{l}\text { PGWB/General } \\
\text { health }\end{array}$ & $-0.323^{*}$ & $-0.231^{*}$ & $-0.284^{*}$ & $-0.193^{*}$ & $-0.241^{*}$ & $-0.271^{*}$ & $-0.341^{*}$ & $-0.323^{*}$ & -0.169 * & $-0.222^{*}$ \\
\hline PGWB/Vitality & $-0.324^{*}$ & $-0.281^{*}$ & $-0.189 *$ & $-0.345^{\star}$ & -0.135 & $-0.138 *$ & -0.260 * & $-0.275^{\star}$ & $-0.173^{*}$ & $-0.183^{*}$ \\
\hline
\end{tabular}

Legend: *statistically significance, $\mathrm{p}<0.05$

Table 6. Correlations of DES and SF-36 Health Survey dimensions scores by respondents' school type. Correlations are express using Kendall's Tau C test.

\begin{tabular}{|c|c|c|c|c|c|c|c|c|c|c|}
\hline & \multicolumn{2}{|c|}{$\begin{array}{l}\text { DES/ Academic } \\
\text { performance }\end{array}$} & \multicolumn{2}{|c|}{$\begin{array}{c}\text { DES/ Difficulties } \\
\text { and insecurities } \\
\text { about professional } \\
\text { future }\end{array}$} & \multicolumn{2}{|c|}{$\begin{array}{c}\text { DES/ } \\
\text { Responsibilities } \\
\text { with patients }\end{array}$} & \multicolumn{2}{|c|}{$\begin{array}{c}\text { DES/ } \\
\text { Personal and } \\
\text { institutional } \\
\text { factors }\end{array}$} & \multicolumn{2}{|c|}{$\begin{array}{l}\text { DES/ } \\
\text { Interpersonal } \\
\text { relationships }\end{array}$} \\
\hline & Public & Private & Public & Private & Public & Private & Public & Private & Public & Private \\
\hline $\begin{array}{l}\text { SF-36/Physical } \\
\text { function }\end{array}$ & $-0.227^{\star}$ & $-0.151^{*}$ & -0.108 & $-0.156^{*}$ & -0.131 & $-0.197^{\star}$ & -0.220 * & $-0.192^{*}$ & -0.047 & $-0.151^{*}$ \\
\hline $\begin{array}{l}\text { SF-36/Role } \\
\text { physical }\end{array}$ & -0.056 & $-0.171^{*}$ & 0.004 & -0.115 & -0.085 & -0.073 & $-0.170 *$ & -0.194 & $-0.232^{\star}$ & 0.001 \\
\hline $\begin{array}{l}\text { SF-36/Bodily } \\
\text { pain }\end{array}$ & $-0.274^{\star}$ & -0.107 & $-0.299 *$ & -0.155 & $-0.249 *$ & -0.082 & $-0.326^{*}$ & -0.132 & -0.124 & $-0.153^{*}$ \\
\hline $\begin{array}{l}\text { SF-36/General } \\
\text { health }\end{array}$ & $-0.245^{*}$ & -0.138 & -0.149 & -0.136 & -0.150 * & -0.094 & $-0.192^{*}$ & -0.164 * & -0.136 & $-0.247^{\star}$ \\
\hline SF-36/Vitality & $-0.368^{\star}$ & -0.209 * & $-0.238^{\star}$ & $-0.194^{*}$ & -0.050 & -0.097 & $-0.227^{\star}$ & $-0.232^{\star}$ & -0.136 & $-0.217 *$ \\
\hline $\begin{array}{l}\text { SF-36/Social } \\
\text { Functioning }\end{array}$ & $-0.207^{\star}$ & $-0.218^{*}$ & $-0.200^{\star}$ & $-0.159 *$ & -0.137 & $-0.142^{\star}$ & $-0.155^{*}$ & -0.349 * & -0.034 & -0.220 * \\
\hline $\begin{array}{l}\text { SF-36/Role } \\
\text { emotional }\end{array}$ & -0.046 & -0.145 & -0.048 & $-0.196 *$ & -0.002 & -0.025 & -0.043 & -0.151 & $-0.188^{*}$ & -0.053 \\
\hline $\begin{array}{l}\text { SF-36/Mental } \\
\text { health }\end{array}$ & $-0.286^{\star}$ & -0.134 & $-0.177^{\star}$ & -0.093 & -0.100 & -0.076 & $-0.274^{*}$ & $-0.162^{\star}$ & $-0.175^{\star}$ & $-0.186^{\star}$ \\
\hline
\end{tabular}

Legend: *statistically significance, $\mathrm{p}<0.05$

between each dimension of DES and SF-36 surveys, according to dental school type, is showed in Table 6 .

DES/academic performance was significantly correlated with all SF-36 dimension, except for SF36/role physical and SF-36/role emotional, in students from public schools. In fact, for those students, SF-36/role emotional was not significantly correlated with 
any DES dimension. Regarding SF-36/bodily pain, almost all DES dimensions showed a significant negative correlation, except for DES/interpersonal relationship.

In students from private schools, the DES/academic performance was significantly correlated with SF-36/physical function, SF-36/role physical, SF-36/vitality, and SF-36/social functioning. These students showed negative significant correlation in SF-36/bodily pain dimension only with DES/interpersonal relationship $(r=-0.153, p=0.024)$.

\section{DISCUSSION}

This study aimed to correlate stressors with psychological well-being and health factors in dental students from both public and private dental schools from the State of Ceará, Brazil. To the best of the authors' knowledge, this the first study to compare the stressors for students in public and private dental schools involving Brazilian students. Overall, students from public dental schools presented higher stressors, as demonstrated in the significantly higher DES total score. Moreover, DES domains were also negatively correlated with psychological well-being and health factors in both public and private dental schools. These correlations presented values ranging from weak to moderate.

Psychological stress is defined as the perceived environmental pressure, which jeopardizes the well-being of an individual ${ }^{14}$. Higher sources of stress are correlated with worst academic performances ${ }^{15}$ and reduced motivation in the academic career ${ }^{16}$. Additionally, the literature also reports that prolonged periods of psychological distress represents a risk factor for burnout, meanwhile strategies to reduce stressors may prevent it in a long term ${ }^{17}$. Regarding dental students, the literature reports prevalence of stress so high as $100 \%{ }^{18}$. Higher emotional and psychological problems are detected in dental students when compared to other health students, such as medical students ${ }^{19}$. It is well established that higher levels of stress are expected in the final years of their education ${ }^{20}$. It is important to highlight that no significant difference was found between public and private students regarding their attendance in clinical/pre-clinical academic courses. Therefore, the differences in the stressors must not be explained by this variable.

The DES instrument is the best tool to assess and quantify stressors, and its Brazilian validated form was used in the present study ${ }^{8}$. This instrument has been largely used in Dentistry, and one systematic review with meta-analysis showed a pooled DES total scores of $2.34(95 \% \mathrm{Cl} 2.22-2.45)$, which suggests the presence of elevated stressors levels in undergraduate dental students ${ }^{21}$. The present study is in accordance with these results, as a similar total mean DES score was found.

Furthermore, an institutional effect in this outcome was shown, since dental students from public schools presented higher scores in comparison to private dental school students. Similar results have been shown in Malaysian dental students, as students from public universities presented higher stressor levels than their counterparts at private dental schools ${ }^{22}$. The explanation of such differences are challenging, and must take into consideration the social background of the students and the institutions involved in this study. Due to the approval of the Federal Law $12.711 / 2012$, which 
determined the reservation of $50 \%$ of the admissions for students coming from public education within every federal university in the country, it can be inferred that students from public schools present lower socioeconomic status when compared to those from private schools. Consequently, it is possible to hypothesize that students attending private universities present less financial related problems, and thus lower stressors. However, no socioeconomic status was assessed in the present study.

In public schools, a great number of paid extracurricular programs or projects are available. However, these activities can also lead to a greater generation of stress. The search for scholarships or other income sources may be intensified in public universities, mainly due to the academic necessities and the need to afford the dwelling expenses or to reduce their financial dependence. On the other hand, the public institutions also offer various opportunities for students getting financial support, such as housing assistance or scholarships for students in socioeconomic vulnerability situation, which may have benefic effect on certain stressors of those students. Additionally, after 2015, these institutions started to offer, for all students, dental materials and instruments throughout the course, in an attempt to decrease school dropout due to problems related to the acquisition of these materials. Since the present study was conducted before this policy, different results could found after the implementation of this new policy.

In Brazil, the National System for Evaluation in Higher Education is responsible for evaluating both private and public higher education institutions, one of these evaluations is composed by the National Student Proficiency exam. This exam has been performed every three years, and it is applied to students in the final years. A previous study showed that public educational institutions presented better ranks when compared to those found for the private ones ${ }^{23}$. More specifically, all public dental schools involved in the present study were evaluated with the best relative positions (score $=5$, the highest score), meanwhile the private institutions presented a mean score of only 2.5. The search for better evaluation by the educational institution can raise the generation of stressors due to the demand for better performance in public school students. In this sense, it is crucial to highlight that the atmosphere created by the faculty, lack of time for relaxation and recreation were the DES domains with the highest effect size in the present study.

The competition to enter in a public dental school is significantly higher in comparison to the selection process in private dental schools. The literature has consistently shown that higher levels of competition may increase the levels of stress ${ }^{24,25}$, which partially explain the results of the present study. Moreover, both public dental schools involved in the present study are financed by the Federal government, which has now been dealing with a major internal crisis.

Despite of the higher overall DES scores found in students from public schools, students from private schools presented a significantly higher scores in four questions of the interpersonal relationships domain. These questions are related to attitudes of school towards women and homosexual dental students, relationship with the opposite sex, and discrimination due to race or class status. This is in agreement with a previous study, which has demonstrated that students in the fourth year from private institution presented the highest total mean score for personal issues compared to 
those from public universities, showing that the personal issue domain may constitute a significant source of stress in private schools ${ }^{22}$.

Dental students are frequently exposed to several stressors, which range from taking tests to enter dental universities, dealing with clinical training, and finally to understand the complex patient-dentist relationship. All these situations can lead to great fatigue, poor health, and development of serious adverse habits such as tobacco smoking, drinking, use and abuse of illicit drugs ${ }^{26}$. Therefore, strategies as meditation; regular practice of physical exercise and sports; acquire a more balanced diet; optimism; sharing feelings and experiences with close people including teachers and classmates have been encouraged ${ }^{26}$. Furthermore, the following measures may be helpful: raising awareness and encouraging dental students to seek out the existent psychological support services.

The total score in the PGWB index was significantly lower in the public dental school students. Additionally, this group showed lower scores in the following domains: anxiety, positive well-being, and vitality. The literature demonstrates that students with a better living situation report a superior general well-being ${ }^{4}$. Additionally, dental students who have claimed to exercise regularly present a higher well-being than their counterparts ${ }^{3}$. These characteristics were not assessed in the present study, and further studies are necessary to establish these conditions.

Regarding health status, assessed by the SF-36, the literature shows that female students and those in the later years of study express more problems $s^{3,4}$. In the present study, the following domains were significantly lower in students from public schools: vitality and social functioning. The age difference among the sample should be put into perspective when interpreting these results.

One of the most remarkable findings of the present study is the significant negative correlation with stressors and several PGWB and SF-36 domains. Previous studies showed similar results in dental students from different nationalities ${ }^{3,4}$. Therefore, it is reasonable to state that high dental students' workload can impair their general health and well-being.

The cross-sectional design, which does not allow temporality, may be the main weakness of the present study. Additionally, a higher external validity of this study may not be expected, as only e-mail and social media were used to recruit the participants. However, a posteriori power calculation showed an estimation of approximately $80 \%$, demonstrating that an appropriate number of dental students were involved in this study.

This is the first study to correlate stressors with psychological well-being and general health in dental students from both public and private school. Furthermore, the use of validated instruments to Portuguese language and the high study power detected are the main strengths of the present study. Within the limits of the present study design, no potential solution may be directly proposed in order to solve the stressors differences and their impact in the overall health and psychological well-being of dental students. Those findings must be used in strategies implementations that may help dental students during their academic careers, especially in improving their coping skills. Additionally, the curriculum planning must consider that stressors may 
be triggered by both academic and non-academic sources. However, it should be put into perspective that eliminating all stressors is almost impossible.

It was concluded that dental students from public and private dental schools presented different stressors pattern. Higher stressors were detected in dental students from public schools. Additionally, most of dental environment sources of stress domains were significantly associated with well-being and general health for both public and private schools.

\section{ACKNOWLEDGMENTS}

This study was supported by the Brazilian Governamental Agency CAPES by using the Portal de Periódicos CAPES. The author Marcelo B. L. Oliveira received a scholarship from Institutional Program of Undergraduate Scientific Research Scholarship (PIBIC) of the Federal University of Ceará during the development of the study. The authors are thankful to the dental schools' deans for the kind support in data collection, and also for Dr. Cassiano Kuchenbecker Rösing and Dr. Roger Keller Celeste for their support in this study. The authors report no other conflict of interest related to the present study.

\section{REFERENCES}

1. Butler G. Definitions of stress. Occas Pap R Coll Gen Pract. 1993 Aug;(61):1-5.

2. Tosevski DL, Milovancevic MP, Gajic SD. Personality and psychopathology of university students. Curr Opin Psychiatry. 2010 Jan;23(1):48-52. doi: 10.1097/YC0.0b013e328333d625.

3. Sugiura G, Shinada K, Kawaguchi Y. Psychological well-being and perceptions of stress amongst Japanese dental students. Eur J Dent Educ. 2005 Feb;9(1):17-25.

4. Uraz A, Tocak YS, Yozgatligil C, Cetiner S, Bal B. Psychological well-being, health, and stress sources in Turkish dental students. J Dent Educ. 2013 Oct;77(10):1345-55.

5. Al-Sowygh ZH. Academic distress, perceived stress and coping strategies among dental students in Saudi Arabia. Saudi Dent J. 2013 Jul;25(3):97-105. doi: 10.1016/j.sdentj.2013.05.002.

6. Cooper CL, Watts J, Kelly M. Job satisfaction, mental health, and job stressors among general dental practitioners in the UK. Br Dent J. 1987 Jan;162(2):77-81.

7. Fonseca E, Rocha C, Kruger E, Tennant M, Mialhe F, Meneghim M. Distribution of dental schools in Brazil. Eur J Gen Dent. 2016 Apr;5(2):47-52. doi: 10.4103/2278-9626.179534.

8. Sangiorgio J, Araujo P, Navarro C, Zen IR, Costa SC, Riberio PHV, et al. Dental Environment Stress: Findings among Lusophone Dental Students. Braz Res Ped Dent Integr Clin. 2016 Dec;16(1):411-24. doi: 10.4034/PBOCI.2016.161.43.

9. Langoski J, Klipan L, Bordin D, Ferracioli M, Pinto M, Fadel C. Stress among Brazilian Dental students in different periods: prevalence and perceptions. Physchol. 2015 Feb;6(3):297-304. doi: 10.4236/psych.2015.63030.

10. Ciconelli R, Ferraz M, Santos W, Meinão I, Quaresma M. Brazilian-Portuguese version of the SF-36. A reliable and valid quality of life outcome measure. Rev Bras Reumatol. 1999 Jan;39(3):143-50.

11. Santo R. [Psychological well-being: validation of Psychological General Well-being scale to portuguese population] [dissertation]. Porto: Faculdade de Psicologia, Educação e Desporto, Universidade Lusófona do Porto; 2015. Portuguese. 
12. Petta CA, Ferriani RA, Abrao MS, et al. Randomized clinical trial of a levonorgestrel-releasing intrauterine system and a depot $\mathrm{GnRH}$ analogue for the treatment of chronic pelvic pain in women with endometriosis. Hum Reprod. 2005 Jul;20(7):1993-8.

13. Davies A, Ware Jr J. Measuring health perceptions in the health insurance experiment. Santa Monica: Rand; 1981.

14. Lazarus R, Folkman S. Stress, appraisal, and coping. New York: Springer; 1984.

15. Sohail N. Stress and academic performance among medical students. J Coll Physicians Surg Pak. 2013 Jan;23(1):67-71. doi: 01.2013/JCPSP.6771.

16. Garbee WH, Zucker SB, Selby GR. Perceived sources of stress among dental students. J Am Dent Assoc. 1980 Jun;100(6):853-7.

17. Awa WL, Plaumann M, Walter U. Burnout prevention: a review of intervention programs. Patient Educ Couns. 2010 Feb;78(2):184-90. doi: 10.1016/j.pec.2009.04.008.

18. Ahmad MS, Md Yusoff MM, Abdul Razak I. Stress and its relief among undergraduate dental students in Malaysia. Southeast Asian J Trop Med Public Health. 2011 Jul;42(4):996-1004.

19. Humphris G, Blinkhorn A, Freeman R, Gorter R, Hoad-Reddick G, Murtomaa H, et al. Psychological stress in undergraduate dental students: baseline results from seven European dental schools. Eur $\mathrm{J}$ Dent Educ. 2002 Feb;6(1):22-9.

20. Gorter R, Freeman R, Hammen S, Murtomaa H, Blinkhorn A, Humphris G. Psychological stress and health in undergraduate dental students: fifth year outcomes compared with first year baseline results from five European dental schools. Eur J Dent Educ. 2008 May;12(2):61-8. doi: 10.1111/j.1600-0579.2008.00468.x.

21. Elani HW, Allison PJ, Kumar RA, Mancini L, Lambrou A, Bedos C. A systematic review of stress in dental students. J Dent Educ. 2014 Feb;78(2):226-42.

22. Babar MG, Hasan SS, Ooi YJ, Ahmed SI, Wong PS, Ahmad SF, et al. Perceived sources of stress among Malaysian dental students. Int J Med Educ. 2015 May 2;6:56-61. doi: 10.5116/ijme.5521.3b2d.

23. Azevedo M, Gomes T, Barbosa R, Barros A, Silva A, Sette-de-Souza P. [Historical series of Enade grades in Dentistry: were there changes throughout three evaluative cycles?] Rev ABENO. 2017;17(1):109-13. Portuguese.

24. Jameson M, Smith J. Voices of students in competition: Health Science First Year at the University of Otago, Dunedin. N Z Med J. 2011 Jul;124(1338):55-67.

25. Bathla M, Singh M, Kulhara P, Chandna S, Aneja J. Evaluation of anxiety, depression and suicidal intent in undergraduate dental students: A cross-sectional study. Contemp Clin Dent. 2015 Apr-Jun;6(2):215-22. doi: 10.4103/0976-237X.156050.

26. Dhama K, Gupta R, Singla A, Patthi B, Ali I, Niraj LK, et al. An Insight into Spiritual Health and Coping Tactics among Dental Students; A Gain or Blight: A Cross-sectional Study. J Clin Diagn Res. 2017 Aug;11(8):ZC33-ZC38. doi: 10.7860/JCDR/2017/25358.10371.

\title{
LIST OF ALL CAPTIONS
}

\author{
Universidade de Fortaleza: UNIFOR
}

Faculdade Católica Rainha do Sertão: FCRS

Dental Environment Stress: DES

Psychological General Well-Being: PGWB

Medical Outcomes Study 36-Item Short-Form Health Survey: SF-36 